\title{
COMPUTATIONAL PARAMETRIC STUDY OF LATENT MELTING HEAT STORAGE
}

\author{
Hamza Faraji*, Mustapha Faraji, Mustapha El Alami
}

LPMMAT Laboratory, Physics Department, Faculty of Sciences Ain Chock, Hassan II University, Casablanca - Morocco

\author{
* Corresponding author: faraji-ham@hotmail.com
}

\begin{abstract}
This paper presents a numerical study of the melting process of a phase change material for the cooling of a heat source "electronic component". The latter is protruding and mounted on a substrate "motherboard" in the center of the bottom wall of a rectangular enclosure. The enclosure is filled completely with PCM. A 2D nondimensional mathematical model is developed based on the conservation equations of mass, momentum and energy. The enclosure aspect ratio ' $A$ ' effect on the thermal and hydrodynamic behavior of the PCM-based heat sink is investigated.
\end{abstract}

Keywords: Melting, PCM, Natural convection, Cooling

\section{Introduction}

The technological advancement known in our era represents an evolution whose miniaturization of electronic cards remains the goal of manufacturers. This miniaturization is accompanied by a high operating frequency which will increase the operating temperature of the electronic chipsets. This increase in temperature is a major cause of failures that can occur on electronic components [1]. Several strategies are known in literature for cooling electronic components and extending their operating life. These strategies are divided into two main axes; the first consists of cooling with ambient air either with natural or forced convection where "ventilation" factor contributes to the cooling process and the second which is concerned with liquid cooling [2-3]. A strategy based on the latent energy storage generated by electronic chipsets using phase change materials (PCMs) has shown an improved efficiency given their high energy storage density. These are PCM-based heat sinks with different configurations [4-5]. The low thermal conductivity of PCMs has prompted several researchers to improve heat transfer within enclosures containing PCMs. This improvement is achieved by metallic structures insertion such as fins with high thermal conductivity, metallic foams or metallic nanoparticles [6-8]. All of these studies search for establishing a more efficient strategy for cooling electronic components. The present study develops a numerical investigation of the melting process of a PCM (n-eicosane) for the cooling of an electronic component in an enclosure completely filled with PCM. The heat source is protruding and mounted on a substrate "motherboard" in the center of the lower wall of the enclosure. The enclosure aspect ratio A effect on the maximal operating temperature of the heat source is investigated numerically.

\section{Analysis and modeling}

The physical model of the studied configuration in this work is shown in Figure 1. Thermo-physical properties of the PCM, the heat source and the substrate are summarized in Table 1.

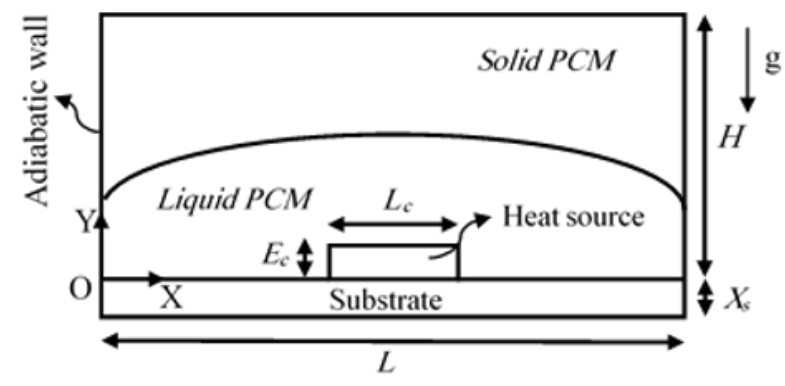

Figure 1 : The physical model

Table 1 : Thermo-physical properties

\begin{tabular}{|l|l|l|}
\hline \multicolumn{1}{|c|}{ Heat source } & \multicolumn{1}{c|}{ PCM (n-eicosane) } & \multicolumn{1}{|c|}{ Substrate } \\
\hline$k_{c}=170 \mathrm{~W} / \mathrm{m} \cdot \mathrm{K}$ & $k_{m}=0.1505 \mathrm{~W} / \mathrm{m} \cdot \mathrm{K}$ & $k_{s}=19.7 \mathrm{~W} / \mathrm{m} \cdot \mathrm{K}$ \\
$\rho_{c}=3260 \mathrm{Kg} / \mathrm{m}^{3}$ & $\rho_{m}=769 \mathrm{Kg} / \mathrm{m}^{3}$ & $\rho_{s}=3900 \mathrm{Kg} / \mathrm{m}^{3}$ \\
$\left(c_{p}\right)_{c}=740 \mathrm{~J} / \mathrm{Kg}$ & $c_{p}=2460 \mathrm{~J} / \mathrm{Kg}$ & $\left(c_{p}\right)_{s}=900 \mathrm{~J} / \mathrm{Kg}$ \\
$\mathrm{T}_{c r}=98{ }^{0} \mathrm{C}$ & $\mu=4.1510^{-2} \mathrm{~m}^{2} / s$ & \\
& $\beta=8.0510^{-4} \mathrm{~K}^{-1}$ & \\
& $\Delta H=2.4710^{5} \mathrm{~J} / \mathrm{Kg}$ & \\
& $\mathrm{T}_{m}=36{ }^{0} \mathrm{C}$ & \\
& & \\
\end{tabular}

The non-dimensional mathematical formulation is based on simplifying assumptions; the PCM is pure, homogeneous and isotropic. The phase change "melting" is considered isothermal. Buoyancy is taken into account by adopting the Boussinesq approximation. The fluid flow is Newtonian, laminar and incompressible.

Continuity equation :

$$
\frac{\partial U}{\partial X}+\frac{\partial V}{\partial Y}=0
$$

Conservation equation of momentum :

$$
\begin{gathered}
\frac{\partial U}{\partial \tau}+\frac{\partial U U}{\partial X}+\frac{\partial V U}{\partial Y}=-\frac{\partial P}{\partial X}+\operatorname{Pr} \frac{\partial}{\partial X}\left(\frac{\partial U}{\partial X}\right)+\operatorname{Pr} \frac{\partial}{\partial Y}\left(\frac{\partial U}{\partial Y}\right)+S_{U} \\
\frac{\partial U}{\partial \tau}+\frac{\partial U V}{\partial X}+\frac{\partial V V}{\partial Y}=-\frac{\partial P}{\partial Y}+\operatorname{Pr} \frac{\partial}{\partial X}\left(\frac{\partial V}{\partial X}\right)+\operatorname{Pr} \frac{\partial}{\partial Y}\left(\frac{\partial V}{\partial Y}\right)+S_{V} \\
\text { with : } \quad S_{U}=-\bar{C} \frac{(1-f)^{2}}{\varepsilon+f^{3}} U+R a \cdot \operatorname{Pr} \cdot \cos (\Omega) \\
S_{V}=-\bar{C} \frac{(1-f)^{2}}{\varepsilon+f^{3}} V+R a \cdot \operatorname{Pr} \cdot \cos (\Omega)
\end{gathered}
$$

Energy equation :

$$
\begin{aligned}
& \quad \frac{\partial \theta}{\partial \tau}+\frac{\partial U \theta}{\partial X}+\frac{\partial V \theta}{\partial Y}=\frac{\partial}{\partial X}\left(\bar{\alpha} \frac{\partial \theta}{\partial X}\right)+\frac{\partial}{\partial Y}\left(\bar{\alpha} \frac{\partial \theta}{\partial Y}\right)+S_{T} \\
& \text { with : } \quad S_{T}=\delta_{1}\left(\left(\delta_{2}-1\right) \frac{1}{\text { Ste }} \frac{\partial f}{\partial \tau}+\frac{Q \delta_{2}}{E_{c} L_{c}}\right)
\end{aligned}
$$

The parameters value $\delta_{1}$ and $\delta_{2}$ depends on the material type (PCM, heat source and substrate). 


\section{Boundary conditions:}

- Adiabatic walls

$$
\left.\frac{\partial \theta}{\partial \eta}\right|_{\text {wall }}=0, \eta \perp \text { wall }
$$

- Interface between two different materials (1,2) (substrate, PCM or electronic component):

$$
-\left.K_{1} \frac{\partial \theta}{\partial \eta}\right|_{\text {interface }}=-\left.K_{2} \frac{\partial \theta}{\partial \eta}\right|_{\text {intefface }}, \theta_{1}=\theta_{2}, \quad \eta \perp \text { wall }
$$

Initial conditions:

$$
\theta=0, \mathrm{U}=\mathrm{V}=0, \mathrm{f}=0
$$

These equations are integrated and discretized on control volumes using the finite volume method [9]. The SIMPLE algorithm is adopted for velocity-pressure coupling. The algebraic equations are solved, for each time step, using the iterative tri-diagonal matrix (TDMA) method. The energy equation for PCM is processed using the enthalpic method [10]. For reasons of brevity, the mathematical model is given in concentrated form.

\section{Results and discussion}

Computational investigations are performed around the PCM "melting" phase change process. The PCM is heated by a heat source acting as a microprocessor and generating a constant volumetric flux density $(\mathrm{Q}=165$ $\left.\mathrm{W} \cdot \mathrm{m}^{-1}\right)$. The enclosure and the heat source characteristic lengths are equal to $l_{0}=0.11 \mathrm{~m}$ and $l_{\mathrm{CE}}=0.012 \mathrm{~m}$, respectively. When varying the enclosure aspect ratio, the PCM amount contained in the enclosure remains constant for all simulations and equal to $1_{0}$. The purpose of this study is to unveil the aspect ratio effect on the heat sink thermal and hydrodynamic behavior. The range of aspect ratios used is spread over $0.25-4$. The value $\mathrm{A}_{\text {ref }}=0.5$ represents the reference aspect ratio.

In this section, we will unveil the aspect ratio effect on the evolution of the heat source maximal temperature, the liquid fraction, the temperature along the substrate at $Y=X_{s} / 2$, the maximal working time of the heat source and the plateau temperature. Figure 2 shows the temporal evolution of the heat source maximal temperature and the liquid fraction. It is clear that the heat transfer is initially conductive given the linear and rapid evolution of temperature for different values of the ratio (A / $\mathrm{A}_{\text {ref }}$ ). The first glimpse of Figure 2 shows that an increase in the aspect ratio results in an increase in the maximal temperature of the heat source and a decrease in the PCM liquid fraction. Indeed, an increase in the aspect ratio causes a decrease in the distance between the heat source and the left and right adiabatic walls. In addition, the more the aspect ratio increases, the more the substrate length decreases by decreasing the substratePCM exchange surface. The decrease of the latter implies that the substrate role for the diffusion of heat produced by the heat source to the PCM will be reduced. This may result in an increase in the heat source temperature because a large amount of heat, generated by the heat source, will be stored by a sensible process in the heat source. We can also observe that the maximal operating time and the long period of the "plateau" region correspond to a higher aspect ratio. Since the minimal of the heat source maximal temperature corresponds to a lower aspect ratio, the configuration with a lower aspect ratio is recommended for cooling purposes of the heat source.

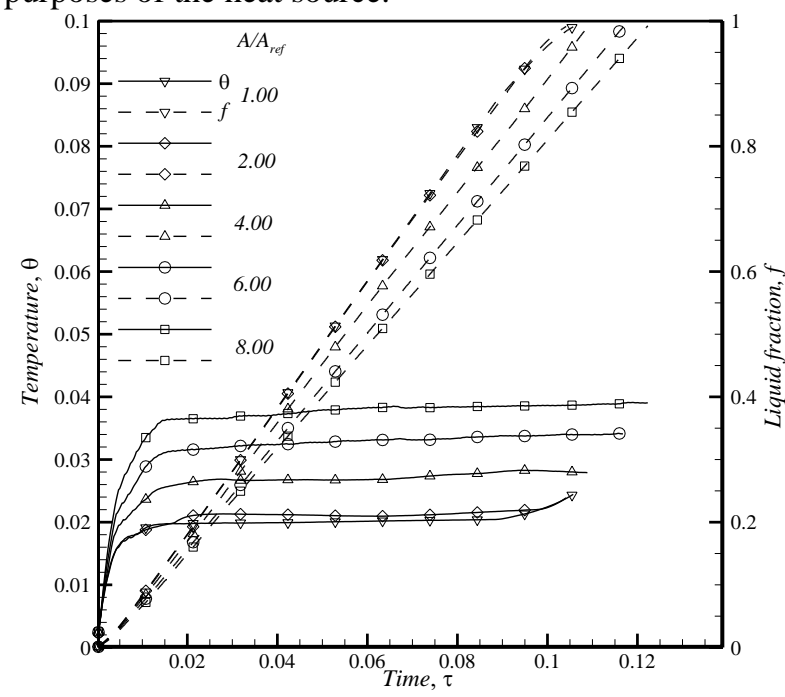

Figure 2: Maximal dimensionless heat source temperature and the liquid fraction evolution

The last result is confirmed by monitoring the temperature evolution along the substrate at $Y=X_{s} / 2$ and $\tau=0.08$ «plateau region ». This evolution is elucidated in Figure 3. It is clear that an increase in the aspect ratio causes an increase in the substrate temperature. The substrate is well cooled for cases of low aspect ratios because it diffuses heat over a large exchange surface with the PCM. On the other hand, for high aspect ratios, the substrate temperature increases. The best contribution of the substrate to the diffusion of heat extracted from the heat source can lead to a cooling of it. If we focus on the temperature difference that can be reached along the substrate, we find that for $A / A_{\text {ref }}=1, \Delta \theta=0.011$ while for $A / A_{\text {ref }}=8, \Delta \theta=0.002$. It is important to note that a large difference in temperature along the substrate gives more opportunity to take place failures. Hence the problem posed, in this study, for the case of low aspect ratios; even if they guarantee a good cooling of the heat source, the risk of failure at the substrate increases.

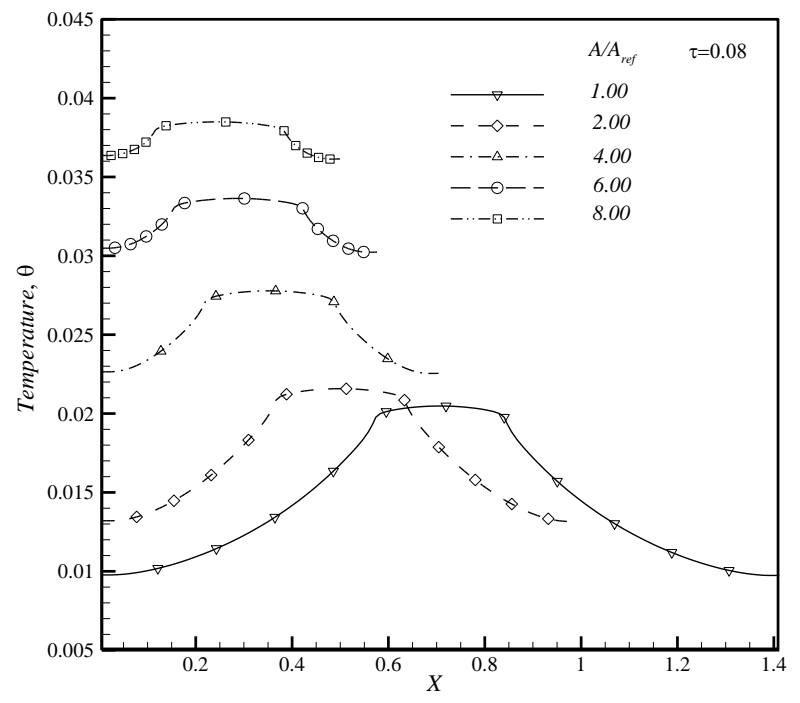

Figure 3 : Substrate temperature profile 
The aspect ratio effect on the heat source maximal operating time and the "plateau" region temperature is illustrated in Figure 4. It is observed that the increase in the ratio $A / A_{\text {ref }}$ from 1 to 8 causes an increase in the heat source maximal operating time and the "plateau" region temperature. For values of $A / A_{\text {ref }}<2$, variations of $\tau_{\max }$ et $\theta_{\text {work }}$ are weak while for values of $A / A_{\text {ref }}>2$, these variations become important.

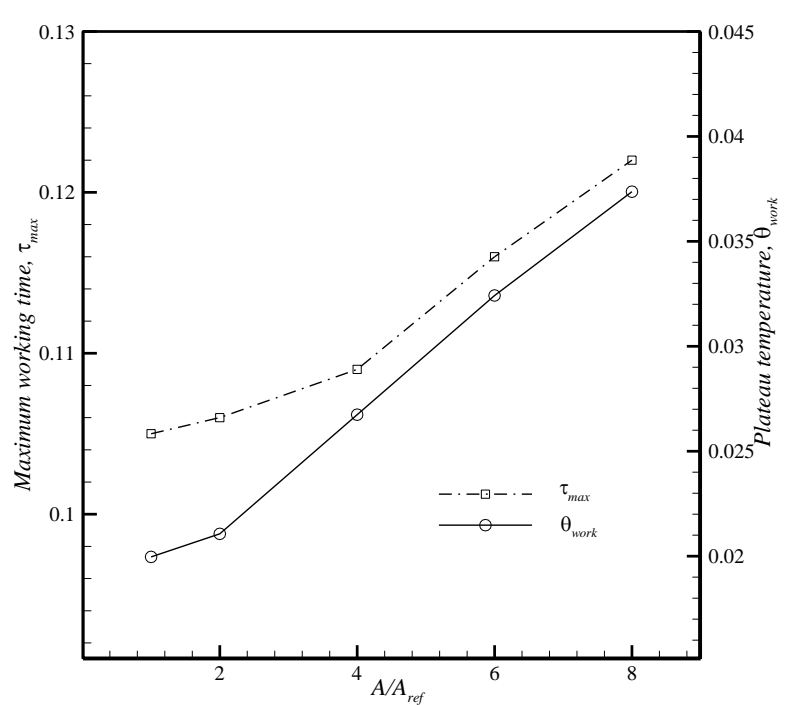

Figure 4 : Maximal operating time and plateau temperature

Based on Figure 4, the heat source maximal operating time and the plateau temperature are correlated by:

$$
\begin{aligned}
& \tau_{\max }=1.040610^{-1}+5.4024110^{-4}\left(\frac{A}{A_{\text {ref }}}\right)+2.174810^{-4}\left(\frac{A}{A_{\text {ref }}}\right)^{2} \\
& \theta_{\text {max }}=1.7210^{-2}+2.2194110^{-3}\left(\frac{A}{A_{\text {ref }}}\right)+4.088910^{-5}\left(\frac{A}{A_{\text {ref }}}\right)^{2}
\end{aligned}
$$

\section{Conclusion}

The melting process of a phase change material for cooling a heat source has been investigated numerically. The enclosure aspect ratio A effect on the evolution of the heat source temperature, the substrate and the liquid fraction is investigated for different values of the ratio $A / A_{\text {ref }}$. Numerical simulations have shown that the more the aspect ratio increases the more the heat source operating temperature and the maximal operating time increase.

\section{References}

[1] L. T. Yeh, Review of Heat Transfer Technologies in Electronic Equipment, Journal of Electronic Packaging 117 (1995).

[2] C. C. Richard, E. S. Robert, J. E. Michael, R. S. Roger and C. Vincent, Review of cooling technologies for computer products, IEEE Transactions on Device and Materials Reliability 4 (2004).
[3] F. P. Incropera, Convection heat transfer in electronic equipment cooling, Journal of Heat Transfer 110 (1988).

[4] J. M. Mahdi, E. C. Nsofor, Solidification enhancement of PCM in a triplex-tube thermal energy storage system with nanoparticles and fins, Journal of Heat Transfer 211 (2018) 975986.

[5] M. Faraji, H. El Qarnia, Numerical study of free convection dominated melting in an isolated cavity heated by three protruding electronic components, IEEE Trans. Components Packag. Technol 33 (2010).

[6] S. Krishnan, S.V. Garimella and S.S. Kang, A novel hybrid heat sink unsing phase change materials for transient thermal management of electronics, IEEE Transact. on Compon. Packging \& Manufact. Technol. 28 (2005) 281289.

[7] W.G. Alshaer, S.A. Nada, M.A. Rady, E. P. Del Barrio and A. Sommier, Thermal management of electronic devices using carbon foam and PCM/nano-composite, International Journal of Thermal Sciences 89 (2015) 79-86.

[8] M. Faraji, Investigation of the melting coupled natural convection of nano phase change material: A fan less cooling of heat sources, FDMP 13 (2017) 19-36.

[9] S. V. Patankar, Numerical Heat Transfer and Fluid Flow, Hemisphere, 1983.

[10] V. R. Voller, M. Cross, N. C. Markatos, An enthalpy method for convection/diffusion phase change, Int. J. for Num. Meth. Engng, 24 (1987) 271-284. 